\title{
Constructal Vascular Structures With High-Conductivity Inserts for Self-Cooling
}

In this paper, we show how a heat-generating domain can be cooled with embedded cooling channels and high-conductivity inserts. The volume of cooling channels and high-conductivity inserts is fixed, so is the volume of the heat-generating domain. The maximum temperature in the domain decreases with high-conductivity inserts even though the coolant volume decreases. The locations and the shapes of high-conductivity inserts corresponding to the smallest peak temperatures for different number of inserts are documented, $x=0.6 \mathrm{~L}$ and $D / B=0.11$ with two rectangular inserts. We also document how the length scales of the inserts should be changed as the volume fraction of the coolant volume over the high-conductivity material volume varies. The high-conductivity inserts should be placed nonequidistantly in order to provide the smallest peak temperature in the heat-generating domain. In addition, increasing the number of the inserts after a limit increases the peak temperature, i.e., this limit is eight number of inserts for the given conditions and assumptions. This paper shows that the overall thermal conductance of a heat-generating domain can be increased by embedding high-conductivity material in the solid domain (inverted fins) when the domain is cooled with forced convection, and the summation of high-conductivity material volume and coolant volume is fixed. [DOI: 10.1115/1.4030906]

Keywords: constructal, vascular, inverted fins, conductive cooling, heat transfer enhancement

\section{Introduction}

Advanced technologies in electronics and avionics require compactness with great volumetric heating rates. For instance, heat generation rate per unit volume in current electronic chips is equivalent to the one in nuclear reactors $[1,2]$. Cooling of electronics became a challenge due to the trend of miniaturization. Miniature designs do not have enough heat transfer surface areas to cool themselves with natural convection and forced convection when the coolant fluid is air. Therefore, forced convection with distinct coolants, such as water, oil and nanofluids, and heat transfer enhancement with extended surfaces, are focused in the current literature [3-11]. In addition, different optimization parameters, such as spacing in between electronic boards and nonuniform chip distribution, are also discussed in the literature [12-17]. However, coolant fluid in convection mechanism always interacts with solid domains. When the thermal resistance of the convective domain is so small in comparison with the thermal resistance of the conductive domain, the rate of the heat transfer is dominated by the conductive thermal resistance. Therefore, increasing the convective heat transfer coefficient after a ceiling value does not change the order of the overall heat transfer rate. The current literature shows that embedding microchannels in the solid domain increases the overall thermal conductance by decreasing the conductive resistances $[18,19]$. These references also show that the pumping power increases with added microchannels and the rate of increase can be decreased by morphing the design.

Furthermore, minimization of the energy consumption in heat exchangers is essential due to two main reasons: scarcity of the

\footnotetext{
${ }^{1}$ Corresponding author.

Contributed by the Heat Transfer Division of ASME for publication in the Journal of Heat Transfer. Manuscript received November 21, 2014; final manuscript received June 15, 2015; published online July 14, 2015. Assoc. Editor: Oronzio Manca.
}

energy sources and global warming. Therefore, finding the best performing systems with minimum energy usage is a necessity. For example, International Space Station has photovoltaic cells which are located as two wings of length scales of $115 \mathrm{ft} \times 38 \mathrm{ft}$ for electricity generation [20]. These cells generate the energy required for the activities of the space station and the conditioning required for the survival of the astronauts.

One of the available theories to uncover the optimal shape of a design is "constructal theory." This theory shows how the shape should be morphed freely in order to minimize resistances in a flow system (flow of heat, fluid and/or stress) $[16,17,21,22]$. Constructal theory is used in distinct fields, such as engineering, biology, geology, and physics $[17,18,21-30]$. In addition, this theory shows the connection between these fields by uncovering how the designs evolve in time in order to minimize resistances, and how the designs in different fields look alike because their designs are governed by the same objective, i.e., design of river-deltas, human lungs, Eiffel Tower, lightning bolts, and trees has the same objective: minimization of resistances to the flow of fluid, heat, and stress [18,31].

Almogbel and Bejan [32] showed that embedding highconductivity materials in heat-generating domains increases the overall thermal conductance of the domains. In addition, literature also shows how thermal conductance of a domain can be maximized with distinct high-conductivity inserts: tree-shaped ( $T$ - and $Y$-shaped) and two- and three-dimensional with and without nonuniform heating [2,32-38]. In the literature, tree-shaped highconductivity materials are embedded in heat-generating domains, and their stems are assumed to be a heat sink at prescribed temperature. However, constant stem temperature would be impossible to implement when the heat-generating domains are elemental domains, i.e., a part of a system which consists of many number of identical elemental domains. Therefore, current literature lacks of commenting on how to carry heat from the stem of the highconductivity insert to a coolant fluid. Unlike in the literature, this 
paper discusses how to use high-conductivity inserts in palpable devices by considering the boundary conditions of real systems.

\section{Model}

Consider a rectangular domain $(L \times H)$, which is heated volumetrically with an embedded channel of diameter $D_{\text {ch }}$ along the width of the domain in which coolant fluid flows, Fig. 1 (top). Coolant fluid enters with $V_{\text {avg }}$ average velocity and $T_{\text {ref }}$ inlet temperature, and it leaves the channel with $T_{\text {out }}$ outlet temperature. The heat conductivity of the solid domain is $k_{1}$. In addition, highconductivity fins of thermal conductivity $k_{\mathrm{h}}$ are embedded in the solid domain in order to decrease the peak temperature in the heat-generating domain as shown in Fig. 1 (bottom). The summation of the cooling channel area and the high-conductivity insert area is fixed, so is the heat-generating solid area. Their ratio is defined as $\phi$ volume fraction. The stems of the high-conductivity fins are attached on the interface of the coolant channel. In addition, the boundaries of the heat-generating domain are symmetry boundaries because the rectangular domain discussed in this paper is an elemental area of a greater domain consists of multiple elemental domains.

The fluid flow is governed by the mass conservation and momentum equations for incompressible steady flow in a twodimensional coordinate system with constant properties and single phase

$$
\begin{gathered}
\frac{\partial u}{\partial x}+\frac{\partial v}{\partial y}=0 \\
u \frac{\partial u}{\partial x}+v \frac{\partial u}{\partial y}=-\frac{1}{\rho} \frac{\partial P}{\partial x}+\nu \nabla^{2} u \\
u \frac{\partial v}{\partial x}+v \frac{\partial v}{\partial y}=-\frac{1}{\rho} \frac{\partial P}{\partial y}+\nu \nabla^{2} v
\end{gathered}
$$

Here, $x$ and $y$ are the spatial coordinates, $u$ and $v$ are the velocity components corresponding to these coordinates, and $P, \nu$, and $\rho$ are the pressure, kinematic viscosity, and fluid density.
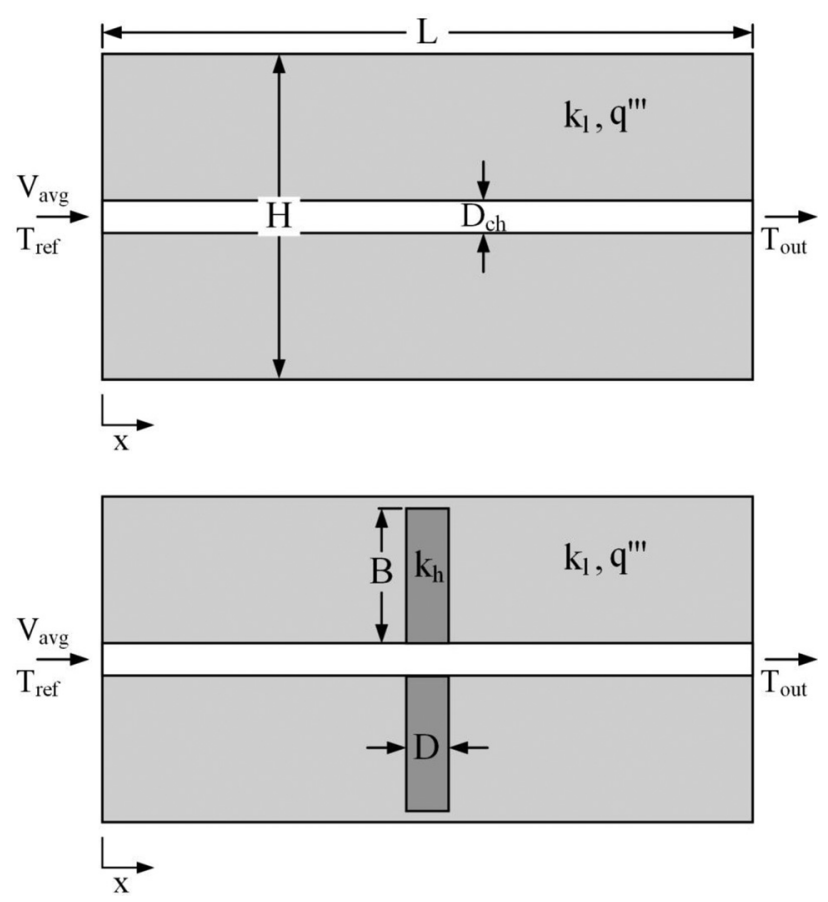

Fig. 1 Heat-generating domain cooled by embedded channel in which coolant fluid flow without high-conductivity inserts (top) and with high-conductivity inserts (bottom)
The temperature distribution inside the cooling channels is governed by the energy equation for fluid domain

$$
\rho c_{\mathrm{P}}\left(u \frac{\partial T}{\partial x}+v \frac{\partial T}{\partial y}\right)=k_{\mathrm{f}} \nabla^{2} T
$$

where $c_{\mathrm{p}}, T$, and $k_{\mathrm{f}}$ are the specific heat at constant pressure of the fluid, temperature, and fluid thermal conductivity, respectively.

The governing equations for the low-conductivity and highconductivity solid domains are

$$
\begin{gathered}
k_{\mathrm{l}} \nabla^{2} T=q^{\prime \prime \prime} \\
k_{\mathrm{h}} \nabla^{2} T=0
\end{gathered}
$$

where $q^{\prime \prime \prime}$ is the volumetric heat rate and $\nabla^{2}=\partial^{2} / \partial x^{2}+\partial^{2} / \partial y^{2}$. The continuity of heat flux between the high-conductivity and low-conductivity solids and fluid interfaces requires

$$
k_{1} \frac{\partial T}{\partial n}=k_{\mathrm{h}} \frac{\partial T}{\partial n} \quad k_{1} \frac{\partial T}{\partial n}=k_{\mathrm{f}} \frac{\partial T}{\partial n} \quad k_{\mathrm{h}} \frac{\partial T}{\partial n}=k_{\mathrm{f}} \frac{\partial T}{\partial n}
$$

where $n$ is the vector normal to the fluid-solid or highconductivity solid-low-conductivity solid interface.

Equations (1)-(3) are nondimensionalized by using $L$ as the length scale and constructing dimensionless velocities in the form of Reynolds numbers

$$
(\tilde{x}, \tilde{y}, \tilde{z}, \tilde{n})=(x, y, z, n) / L, \quad \tilde{u}=u L / \nu, \quad \tilde{v}=v L / \nu, \quad \tilde{w}=w L / \nu
$$

The dimensionless pressure difference is defined as Bejan number $[39,40]$

$$
\tilde{P}=\frac{\left(P-P_{\text {out }}\right) L^{2}}{\mu \alpha}
$$

where $P, P_{\text {out }}, \mu$, and $\alpha$ are the local pressure, outlet pressure, dynamic viscosity, and thermal diffusivity of the fluid $\left(\alpha=k_{\mathrm{f}} / \rho c_{\mathrm{p}}\right)$. The dimensionless temperature and continuity of the heat flux on solid-fluid and high-conductivity solid-low-conductivity solid interfaces are indicated by

$$
\begin{gathered}
\tilde{T}=\frac{\left(T-T_{\mathrm{ref}}\right) k_{\mathrm{f}}}{q^{\prime \prime \prime} L^{2}} \\
\left.5 \tilde{k} \frac{\partial \tilde{T}}{\partial \tilde{n}}\right|_{\mathrm{h}}=\left.\left.\frac{\partial \tilde{T}}{\partial \tilde{n}}\right|_{1} \quad \tilde{k} \frac{\partial \tilde{T}}{\partial \tilde{n}}\right|_{1}=\left.\left.\frac{\partial \tilde{T}}{\partial \tilde{n}}\right|_{\mathrm{f}} 100 \tilde{k} \frac{\partial \tilde{T}}{\partial \tilde{n}}\right|_{\mathrm{h}}=\left.\frac{\partial \tilde{T}}{\partial \tilde{n}}\right|_{\mathrm{f}}
\end{gathered}
$$

where $T_{\text {ref }}$ is the fluid inlet temperature and $\tilde{k}=k_{1} / k_{\mathrm{f}}$. The coefficients of 5 and 100 appear in Eq. (11), because the ratio of the thermal conductivities is fixed at $k_{\mathrm{h}} / k_{\mathrm{l}}=100$ and $k_{1} / k_{\mathrm{f}}=20$. The solutions hold for the entire family of materials which are conforming these ratios; one example could be water, stainless steel, and graphite combination. The solution procedure was also repeated for material combination of engine oil, aluminum, and graphene, which also ensures $15 \%$ decrease in the peak temperature but these solutions were not discussed in details due to the complexity of graphene production.

The dimensionless mass conservation and momentum equations are

$$
\begin{gathered}
\frac{\partial \tilde{u}}{\partial \tilde{x}}+\frac{\partial \tilde{v}}{\partial \tilde{y}}=0 \\
\tilde{u} \frac{\partial \tilde{u}}{\partial \tilde{x}}+\tilde{v} \frac{\partial \tilde{u}}{\partial \tilde{y}}=-\frac{1}{\operatorname{Pr}} \frac{\partial \tilde{P}}{\partial \tilde{x}}+\tilde{\nabla}^{2} \tilde{u}
\end{gathered}
$$




$$
\tilde{u} \frac{\partial \tilde{v}}{\partial \tilde{x}}+\tilde{v} \frac{\partial \tilde{v}}{\partial \tilde{y}}=-\frac{1}{\operatorname{Pr}} \frac{\partial \tilde{P}}{\partial \tilde{y}}+\tilde{\nabla}^{2} \tilde{v}
$$

where Pr is the Prandtl number and $\tilde{\nabla}^{2}=\partial^{2} / \partial \tilde{x}^{2}+\partial^{2} / \partial \tilde{y}^{2}$. The dimensionless energy equation is

$$
\operatorname{Pr}\left(\tilde{u} \frac{\partial \tilde{T}}{\partial \tilde{x}}+\tilde{v} \frac{\partial \tilde{T}}{\partial \tilde{y}}+\tilde{w} \frac{\partial \tilde{T}}{\partial \tilde{z}}\right)=\nabla^{2} \tilde{T}
$$

The dimensionless governing equations of Eqs. (5) and (6) are

$$
\begin{aligned}
\tilde{k} \tilde{\nabla}^{2} \tilde{T} & =1 \\
\tilde{\nabla}^{2} \tilde{T} & =0
\end{aligned}
$$

\section{Numerical Method}

Consider the two-dimensional domain $(L \times H)$ with a channel placed in the middle along which coolant at reference temperature $\left(T_{\text {ref }}\right)$ flows with $V_{\text {avg }}$ inlet velocity (Fig. 1). Volumetric heating rate on the domain of $k_{1}$ thermal conductivity is $q^{\prime \prime \prime}$. Highconductivity material with height of $B$ and thickness of $D$ is embedded on the solid domain as shown in Fig. 1 (bottom). Dimensionless inlet average velocity is 2000, Prandtl number is 6 , $H / L=1, D / B=0.15$, and $\phi=0.1$ in the numerical simulations.

The dimensionless mass conservation, momentum, and energy equations were solved by using a FINITE ELEMENT software [41]. The grid was nonuniform in $\tilde{x}$ and $\tilde{y}$ directions. Boundary layer meshes are also used at the interfaces in order to minimize the numerical errors due to variation in the temperature gradients. The mesh size was determined by increasing the number of the mesh elements until the criterion $\left|\left(\tilde{T}_{\max }^{n}-\tilde{T}_{\max }^{n+1}\right) / \tilde{T}_{\max }^{n}\right|$ $<2 \times 10^{-2}$ was satisfied. $\tilde{T}_{\max }^{n}$ and $\tilde{T}_{\max }^{n+1}$ represent the maximum dimensionless temperature by using the current mesh and the refined mesh, respectively. The residual was imposed as $10^{-8}$ in the numerical software. Table 1 illustrates that mesh independency was achieved with 2358 number of mesh elements for the design shown in Fig. 1 (bottom). In addition, the overall heat transfer rate on the stem surface of the high-conductivity insert $\left(q_{\text {num }}^{\prime}\right)$ is compared with the heat transfer rate calculated from the heat generation rate $\left(q_{\mathrm{imp}}^{\prime}\right)$ as shown in Table 1 . The error in between the generated heat and the transferred heat was found as $0.5 \%$ for the selected mesh size. This shows that the first law of thermodynamics is also satisfied.

The numerical results obtained by using the current method have been compared with the numerical results of Almogbel and Bejan [32] for the validation of the numerical method. The dimensionless governing equations of Ref. [32] was solved with the given geometry and boundary conditions when $\varphi=0.1$, $\mathrm{H} / L=1$, and $\mathrm{D} / B=0.15$. Table 2 shows that the results of the current numerical method agree with the results of Ref. [32].

\section{High-Conductivity Inserts}

Consider the high-conductivity inserts with thermal conductivity of $k_{\mathrm{h}}$ and with length scales of $D$ and $B$ embedded in a heat-

Table 1 Numerical tests showing that the result of the simulations does not depend on the mesh size

\begin{tabular}{lccl}
\hline \hline $\begin{array}{l}\text { Number } \\
\text { of elements }\end{array}$ & $\tilde{T}_{\max }^{n}$ & $\left|\left(q_{\text {imp }}^{\prime}-q_{\text {num }}^{\prime}\right) / q_{\text {imp }}^{\prime}\right|$ & $\left|\left(\tilde{T}_{\max }^{n}-\tilde{T}_{\max }^{n+1}\right) / \tilde{T}_{\max }^{n}\right|$ \\
\hline 352 & 0.0072874 & 0.036878 & \\
484 & 0.0072984 & 0.035711 & 0.00151 \\
928 & 0.0068289 & 0.026822 & 0.064329 \\
1336 & 0.0069604 & 0.016389 & 0.01926 \\
2358 & 0.0068323 & 0.005678 & 0.018404 \\
\hline \hline
\end{tabular}

Table 2 Comparison of the results of the current study and Ref. [32]

\begin{tabular}{lllc}
\hline \hline$\tilde{k}$ & $\tilde{T}_{\max }(\mathrm{FE})^{\mathrm{a}}$ & $\tilde{T}_{\max }(\mathrm{FD})^{\mathrm{a}}$ & $\tilde{T}_{\max }(\mathrm{Comsol})^{\mathrm{b}}$ \\
\hline 1000 & 0.128236 & 0.128979 & 0.12847 \\
300 & 0.135924 & 0.135408 & 0.13616 \\
100 & 0.157219 & 0.152994 & 0.15745 \\
30 & 0.224812 & 0.218397 & 0.22509 \\
10 & 0.374893 & 0.37539 & 0.37559 \\
\hline \hline
\end{tabular}

${ }^{a}$ Reference [32].

${ }^{\mathrm{b}}$ Current study.
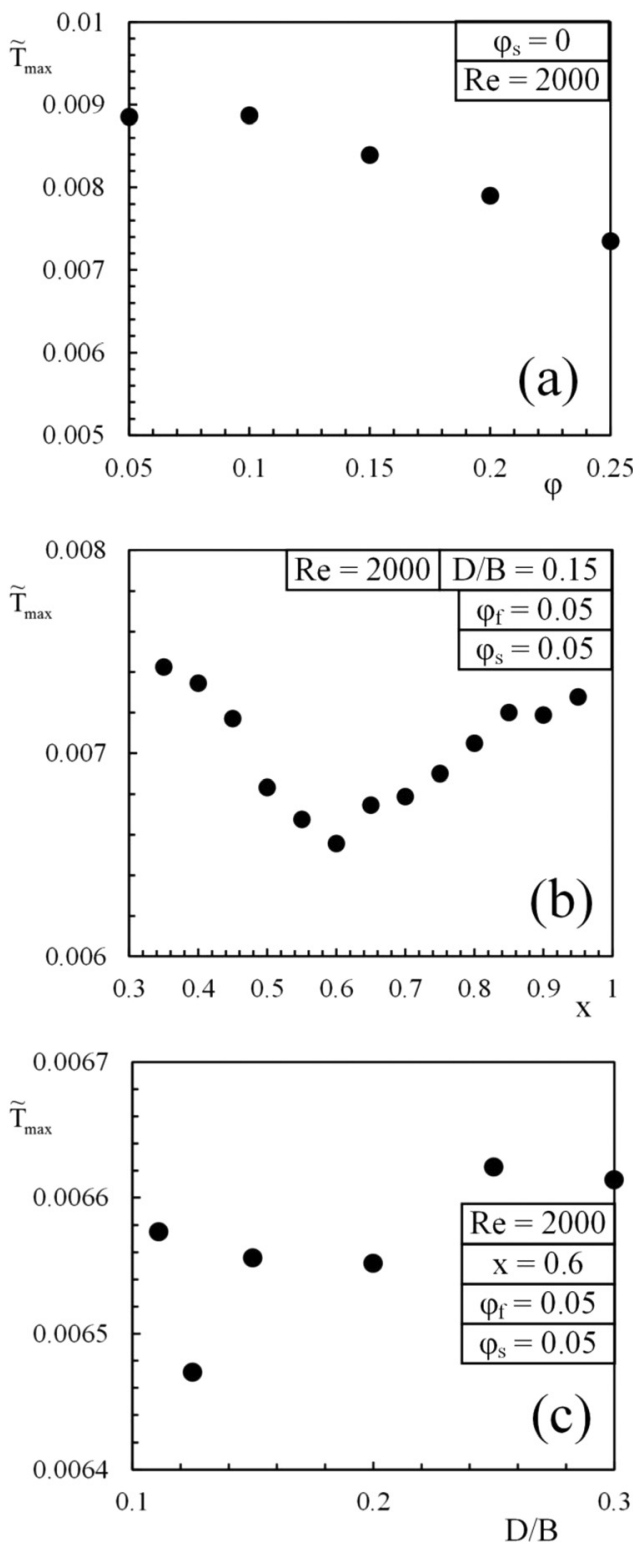

Fig. 2 (a) The effect of coolant volume fraction on maximum temperature without high-conductivity inserts. (b) The effect of the location of the high-conductivity inserts on maximum temperature. (c) The effect of the shape of the inserts on maximum temperature.

generating domain. This domain is cooled by a coolant fluid which flows along the domain as shown in Fig. 1 (bottom). The heat-generating domain is an elemental part of a greater domain. Figure 2(a) shows that how $\tilde{T}_{\max }$ is affected by $\varphi$ (coolant volume/heat-generating domain volume) when the elemental 
domain is solely cooled by convection via flowing coolant along the channel with $\operatorname{Re}=2000 . \tilde{T}_{\max }$ decreases as $\varphi$ increases. However, as $\varphi$ increases the heat-generating domain volume decreases, i.e., the rate of total heat generation decreases.

Figure $2(b)$ shows how the location of the high-conductivity insert affects $\tilde{T}_{\max }$ when both $\varphi_{\mathrm{f}}$ (coolant fluid volume/heatgenerating domain volume) and $\varphi_{\mathrm{s}}$ (high-conductivity material volume/heat-generating domain volume) are fixed at 0.05 . The summation of the coolant volume and high-conductivity material volume over the heat-generating domain is $\varphi=\varphi_{\mathrm{s}}+\varphi_{\mathrm{f}}=0.1$. Comparison of Figs. 2(a) and 2(b) for $\varphi=0.1$ shows that embedding high-conductivity inserts decreases $\tilde{T}_{\max }$ from 0.009 to 0.0076-0.0064 range for a specific $x$ location of the highconductivity insert. This comparison shows that embedding high-conductivity inserts increases the overall thermal conductance of a heat-generating domain. Therefore, using hybrid of high-conductivity inserts (i.e., high-conductivity fins inserted in solid material) and convective cooling ensures greater cooling performance in comparison with sole convective cooling. In addition, Fig. $2(c)$ shows how $D / B$ ratio affects $\tilde{T}_{\max } \cdot \tilde{T}_{\max }$ is the smallest with $D / B=0.11$ when $\operatorname{Re}=2000, x=0.6$, and $\varphi_{\mathrm{s}}=\varphi_{\mathrm{f}}=0.05$.

Figure 3(a) shows how Reynolds number (i.e., dimensionless average inlet velocity) affects the peak temperature with and without high-conductivity inserts as shown in Fig. 1. The effect of inserting high-conductivity inserts is negligibly small in comparison with the peak temperature when $\mathrm{Re}=200$ because the generated heat is greater than the cooling capability of the coolant fluid. Therefore, the rate of decrease in the peak temperature increases when $\operatorname{Re}>200$ with high-conductivity inserts. In addition, the return of increasing $\mathrm{Re}$ number decreases as $\mathrm{Re}$ increases, i.e.,
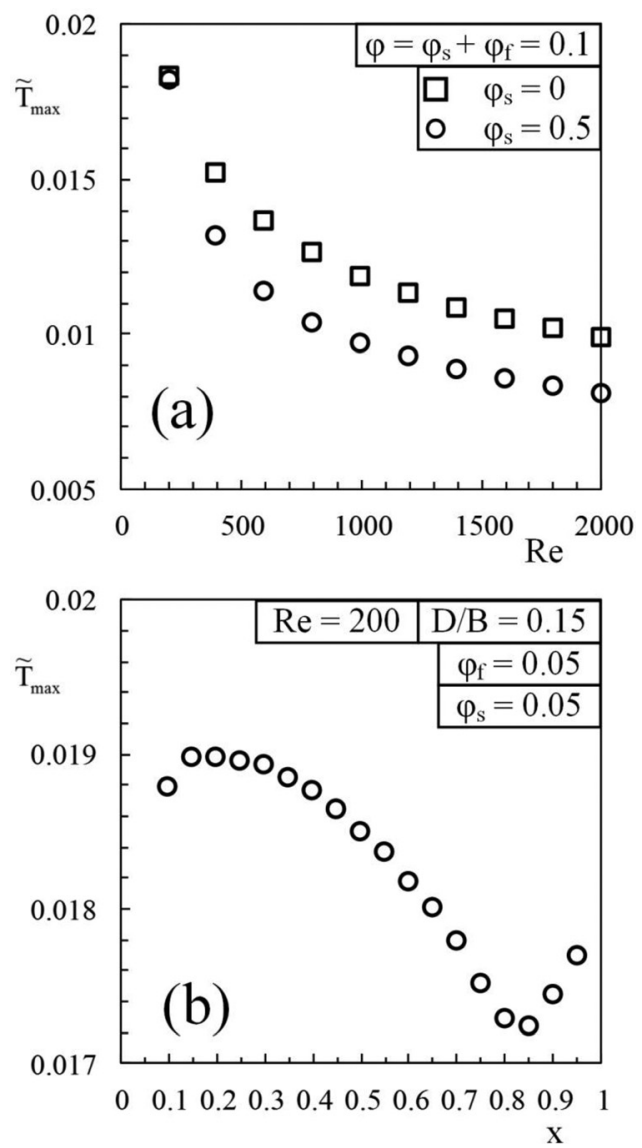

Fig. 3 (a) The effect of Re number on peak temperature with and without high-conductivity inserts. (b) The effect of the location of the high-conductivity inserts on peak temperature with $\mathrm{Re}=200$. the order of the peak temperature converges to the asymptote. Figure $3(b)$ shows how the location of the high-conductivity inserts along the $x$ direction affects the peak temperature when $\operatorname{Re}=200$. The trend of Fig. 3(b) is similar to the trend of Fig. 2(b), however, the $x$ location which provides the smallest peak temperature is moved from $x=0.6$ to $x=0.85$ as $\mathrm{Re}$ decreases from 2000 to 200.

Figure 4 shows how $V_{\mathrm{f}} / V_{\mathrm{h}}$ (volume of the coolant fluid/volume of the high-conductivity material) affects $\tilde{T}_{\max }$ for variable $D / B$ values for the design shown in Fig. 1 (bottom). As $V_{\mathrm{f}} / V_{\mathrm{h}}$ increases from 0.25 to 1 , the effect of varying $D / B$ ratio on $\tilde{T}_{\max }$ diminishes, i.e., $2 \%$ maximum. The effect of $D / B$ ratio on $\tilde{T}_{\max }$ becomes up to $7 \%$ as $V_{\mathrm{f}} / V_{\mathrm{h}}$ becomes 4 . In addition, as $V_{\mathrm{f}} / V_{\mathrm{h}}$ increases from 1 to $4 \tilde{T}_{\max }$ is the minimum with $D / B=0.125$. However, there is an $D / B$ value which corresponds to minimum $\tilde{T}_{\max }$ when $V_{\mathrm{f}} / V_{\mathrm{h}}<1$. Note that for $V_{\mathrm{f}} / V_{\mathrm{h}}=0.25, D / B$ value cannot be taken less than 0.2 because high-conductivity inserts penetrate the walls of the heat-generating domain when $D / B<0.2$. Moreover, $\tilde{T}_{\max }$ is minimum when $V_{\mathrm{f}} / V_{\mathrm{h}}=0.5$, i.e., high-conductivity material volume is two times the coolant volume.

\section{Multiple Inserts}

Consider next the heat-generating domain with two levels of high-conductivity inserts which are placed at locations $x_{1}$ and $x_{2}$ as shown in Fig. 5. Figure 5 shows how $\tilde{T}_{\max }$ is affected by the location of $x_{2}$ when $x_{1}$ is fixed. $\tilde{T}_{\max }$ is the minimum when $x_{1}=0.8$ and $x_{2}=0.35$. Figure 5 also shows that the highconductivity inserts should not be distributed equidistantly in the heat-generating domain which is similar to the results of Fig. 2(b), i.e., $T_{\max }$ is the minimum with $x=0.6$ for one level of insert. The reason of this asymmetry is due to nonhomogeneous temperature distribution along the heat-generating domain. The temperature is the maximum at the top and bottom corners of the heat-generating domain at $x=L$. Therefore, the overall thermal conductance of the heat-generating domain can be increased by moving highconductivity inserts closer to the fluid outlet. However, conductive thermal resistance of the other end increases as the inserts move closer to the fluid outlet. Therefore, there is an optimum location for high-conductivity materials to be placed, i.e., $x=0.6$ for one insert and $x_{1}=0.8$ and $x_{2}=0.35$ for two inserts with $\mathrm{Re}=2000$.

Figure 6 shows how $B / H$ and the locations of the highconductivity inserts, $x_{i}$, affect $\tilde{T}_{\max }$ when three levels of inserts are embedded. First, consider that these three inserts distributed equidistantly in the domain, $x_{1}, x_{2}$, and $x_{3}=0.25,0.5$, and 0.75 , respectively, as shown with filled circles in Fig. $6 . \tilde{T}_{\max }$ is the minimum with $B / H=0.4$ when the three inserts are distributed equidistantly in the domain. The ratio of $B / H$ is used rather than $D / B$ ratio because the results show that the thickness of the

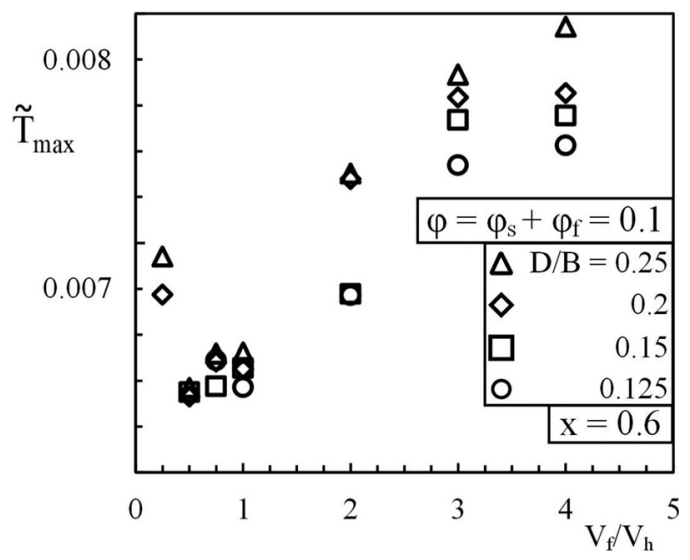

Fig. 4 The effect of the ratio of coolant volume to highconductivity material volume on maximum temperature for $D / B=0.125,0.15,0.2$, and 0.25 


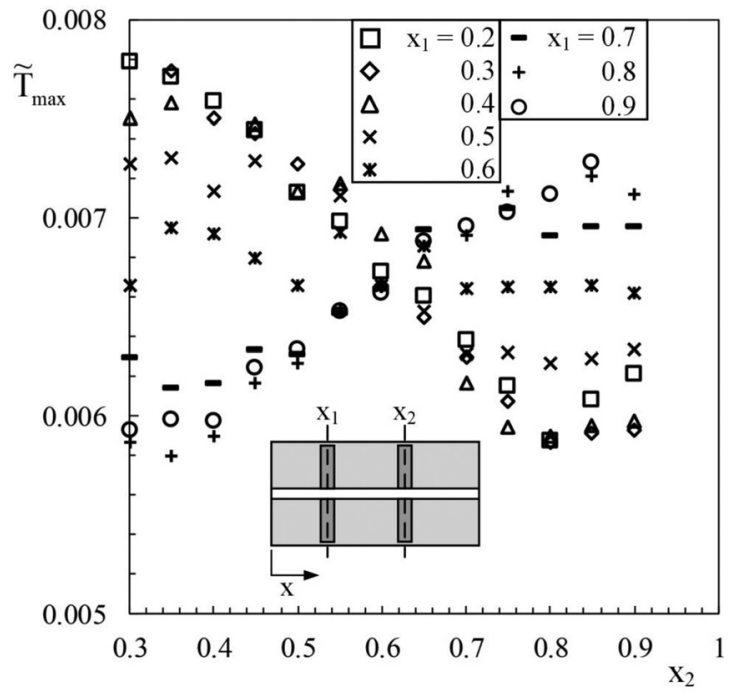

Fig. 5 The effect of the location of the second level of high-conductivity inserts on maximum temperature when the location of the first level of inserts is fixed

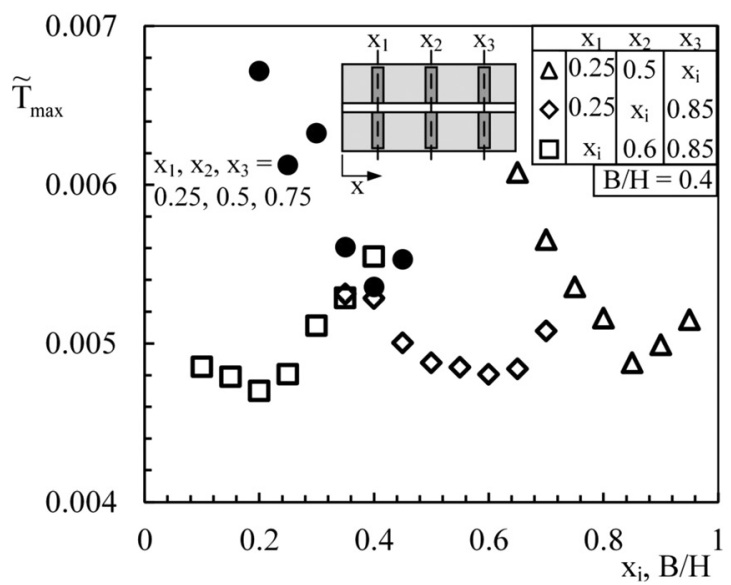

Fig. 6 The effect of the location of the high-conductivity inserts on $\tilde{T}_{\max }$ when $B / H=0.4$ and $\phi_{f}=\phi_{s}=0.05$, and the effect of $B / H$ length scale on $\tilde{T}_{\max }$ when the inserts are placed in the domain equidistantly with $\phi_{f}=\phi_{s}=0.05$

high-conductivity insert $(D)$ does not affect the peak temperature as its height $(B)$ does. Then, consider how the locations of these inserts affect $\tilde{T}_{\max }$ when $B / H=0.4$, which corresponds to the minimum peak temperature for three equidistant inserts. First, $x_{1}$ and $x_{2}$ were fixed at the locations of 0.25 and 0.5 , and the effect of varying $x_{3}$ on $\tilde{T}_{\max }$ is uncovered. $\tilde{T}_{\max }$ is the minimum with $x_{3}=0.85$. Next, $x_{1}$ and $x_{3}$ were fixed at 0.25 and 0.85 (note that $x_{3}=0.85$ is the optimized value of $x_{3}$ while $x_{1}$ and $x_{2}$ were fixed), and the optimum location for $x_{2}$ was found as 0.6 . This procedure is repeated for $x_{1}$, and $\tilde{T}_{\max }$ becomes the minimum when highconductivity inserts are located at $x_{1}=0.2, x_{2}=0.6$, and $x_{3}=0.85$.

Figure 7 shows how $B / H$ affects $\tilde{T}_{\max }$ with multiple levels of high-conductivity inserts. Each level has two fins: one placed in the top part of the domain and the other placed in the bottom part. Figure 7 also shows that $\tilde{T}_{\max }$ is the minimum with a $B / H$ value which varies based on the number of fins. In addition, $\tilde{T}_{\max }$ is the minimum with eight high-conductivity fins when $B / H=0.3$. This shows that increasing the number of fins decreases $\tilde{T}_{\max }$ up to a limit. Therefore, there is an optimum number of fins for maximizing the thermal conductance of a heat-generating domain. Figure 7 also shows that homogeneous distribution of the

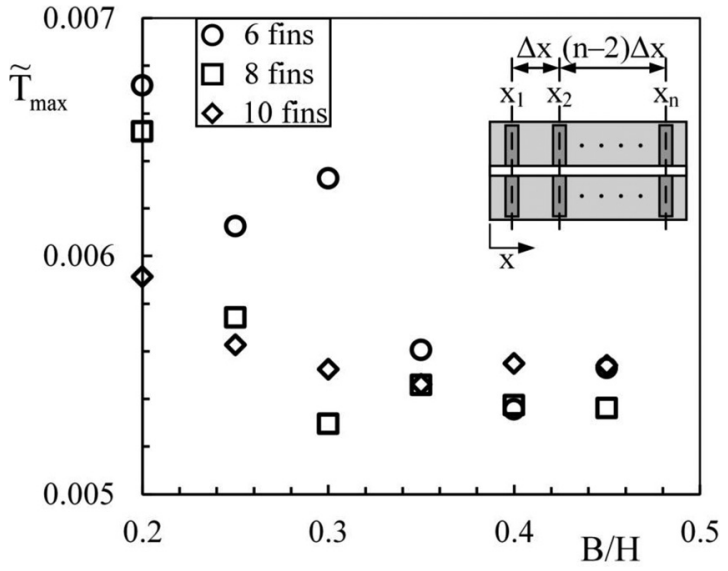

Fig. 7 The effect of $B / H$ length scale on maximum temperature with 6,8 , and 10 equidistant high-conductivity inserts

high-conductivity material in the heat-generating domain does not provide minimum $\tilde{T}_{\max }$, i.e., high-conductivity inserts provide better cooling than homogeneous distribution of high-conductivity material as literature shows [33]. In addition, the quantity of highconductivity material, shape of the inserts, and their numbers and locations are also essential in order to maximize overall thermal conductance of a heat-generating domain.

\section{Conclusions}

This paper shows that the peak temperature of a heatgenerating domain which is cooled with embedded cooling channels can be decreased with inserting high-conductivity material. The volume of the heat-generating domain is fixed, so is the summation of the cooling channels and high-conductivity material volumes. Embedding high-conductivity inserts increases the overall thermal conductance of the heat-generating domain even though coolant volume reduces due to fixed volume constraint. This paper also shows that high-conductivity material should be located a little bit further from the middle and closer to the coolant outlet, namely, $x=0.6$ when $\mathrm{Re}=2000$. In addition, it is showed that as Re decreases the location of the high-conductivity material should be moved closer to the outlet end in order to minimize the peak temperature. These findings are essential because the current literature does not cover the effect of location of the highconductivity inserts on the thermal conductance. In addition, the effect of shape and volume fraction on the peak temperature is also documented.

In addition, this paper shows how to locate and morph highconductivity inserts in order to minimize peak temperature with multiple levels of inserts. Increasing the number of inserts decreases the peak temperature up to eight fins. Further increase in the number of inserts increases the peak temperature. Because of these, it can be concluded that distributing high-conductivity material homogenously into the heat-generating domain does not decrease the peak temperature as embedding inserts with the optimized shapes and locations documented in this paper. Here, we used constructal theory, i.e., the design morphed freely in order to find the design with the smallest resistance, in order to find minimum peak temperature. The high-conductivity material should be inserted such that the overall thermal conductance of the heatgenerating domain becomes the greatest, i.e., as the volume fraction and number of fins change the shape and locations of the fins should be changed. However, the current literature and our previous research show that the designs which correspond to the smallest resistances will change as boundary conditions, constraints, and assumptions vary, i.e., there is no best design for all applications but designs perform the best under given constraints and conditions. 


\section{Acknowledgment}

This work was supported by the Scientific and Technological Research Council of Turkey (TUBITAK) under Grant No. 114M592.

\section{Nomenclature}

$B=$ height of a high-conductivity insert, $\mathrm{m}$

$c_{\mathrm{p}}=$ specific heat at constant pressure, $\mathrm{J} \mathrm{kg}^{-1} \mathrm{~K}^{-1}$

$D=$ thickness of a high-conductivity insert, $\mathrm{m}$

$H=$ height of the conducting domain, m, Fig. 1

$k=$ thermal conductivity, $\mathrm{W} \mathrm{m}^{-1} \mathrm{~K}^{-1}$

$L=$ length scale of the domain, m, Fig. 1

$n=$ normal direction

$P=$ pressure, $\mathrm{N} \cdot \mathrm{m}^{-2}$

$\mathrm{Pr}=$ Prandtl number

$q^{\prime}=$ heat transfer rate per unit length, $\mathrm{W} \mathrm{m}^{-1}$

$q^{\prime \prime \prime}=$ heat source, $\mathrm{W} \mathrm{m}^{-3}$

$T=$ temperature, $\mathrm{K}$

$u, v=$ velocity components, $\mathrm{m} \mathrm{s}^{-1}$

$V=$ inlet velocity, $\mathrm{m} \mathrm{s}^{-1}$

$x, y=$ coordinates, $\mathrm{m}$

\section{Greek Symbols}

$\alpha=$ fluid thermal diffusivity, $\mathrm{m}^{2} \mathrm{~s}^{-1}$

$\phi=$ volume fraction

$\mu=$ dynamic viscosity, $\mathrm{kg} \mathrm{m}^{-1} \mathrm{~s}^{-1}$

$\nu=$ kinematic viscosity, $\mathrm{m}^{2} \mathrm{~s}^{-1}$

$\rho=$ density, $\mathrm{kg} \mathrm{m}^{-3}$

\section{Subscripts}

$$
\begin{aligned}
\text { avg } & =\text { average } \\
\mathrm{f} & =\text { fluid } \\
\mathrm{h} & =\text { high } \\
\mathrm{in} & =\text { inlet } \\
\mathrm{imp} & =\text { imposed } \\
\mathrm{l} & =\text { low } \\
\max & =\text { maximum } \\
\text { num } & =\text { numerical } \\
\text { out } & =\text { outlet } \\
\text { ref } & =\text { reference } \\
\mathrm{s} & =\text { solid }
\end{aligned}
$$

\section{Superscripts}

$n=$ index of the mesh independency test

$\sim=$ dimensionless

\section{References}

[1] Pop, E., Sinha, S., and Goodson, K. E., 2006, "Heat Generation and Transport in Nanometer-Scale Transistors," Proc. IEEE, 94(3), pp. 1587-1601.

[2] Cetkin, E., 2015, "Inverted Fins for Cooling of a Non-Uniformly Heated Domain," J. Therm. Eng., 1(1), pp. 1-9.

[3] Bejan, A., and Ledezma, G. A., 1996, "Thermodynamic Optimization of Cooling Techniques for Electronic Packages," Int. J. Heat Mass Transfer, 39(6), pp. 1213-1221.

[4] Said, S. A. M., 1996, "Investigation of Natural Convection in Convergent Vertical Channels," Int. J. Energy Res., 20(7), pp. 559-567.

[5] Jang, D., Yook, S.-J., and Lee, K.-S., 2014, "Optimum Design of a Radial Heat Sink With a Fin-Height Profile for High-Power Led Lighting Applications," Appl. Energy, 116, pp. 260-268.

[6] Ho, T., Mao, S. S., and Greif, R., 2010, "Improving Efficiency of HighConcentrator Photovoltaics by Cooling With Two-Phase Forced Convection," Int. J. Energy Res., 34(14), pp. 1257-1271.

[7] Kakac, S., and Pramuanjaroenkij, A., 2009, "Review of Convective Heat Transfer Enhancement With Nanofluids," Int. J. Heat Mass Transfer, 52(13-14), pp. 3187-3196.

[8] Eastman, J. A., Choi, S. U. S., Li, S., Yu, W., and Thompson, L. J., 2001 "Anomalously Increased Effective Thermal Conductivities of Ethylene Glycol-Based Nanofluids Containing Copper Nanoparticles," Appl. Phys. Lett., 78(6), pp. 718-720.
[9] Lorenzini, G., Correa, R. L., dos Santos, E. D., and Rocha, L. A. O., 2011 , "Constructal Design of Complex Assembly of Fins," ASME J. Heat Transfer, 133(8), p. 081902.

[10] Bejan, A., and Morega, A. M., 1993, "Optimal Arrays of Pin Fins and Plate Fins in Laminar Forced-Convection," ASME J. Heat Transfer, 115(1), pp. $75-81$.

[11] Lorenzini, G., and Moretti, S., 2011, "Bejan's Constructal Theory Analysis of Gas-Liquid Cooled Finned Modules," ASME J. Heat Transfer, 133(7), p. 071801.

[12] Bejan, A., Fowler, A. J., and Stanescu, G., 1995, "The Optimal Spacing Between Horizontal Cylinders in a Fixed Volume Cooled by Natural Convection," Int. J. Heat Mass Transfer, 38(11), pp. 2047-2055.

[13] da Silva, A. K., Lorente, S., and Bejan, A., 2004, "Optimal Distribution of Discrete Heat Sources on a Wall With Natural Convection,” Int. J. Heat Mass Transfer, 47(2), pp. 203-214.

[14] da Silva, A. K., Lorente, S., and Bejan, A., 2004, "Optimal Distribution of Discrete Heat Sources on a Plate With Laminar Forced Convection," Int. J. Heat Mass Transfer, 47(10-11), pp. 2139-2148.

[15] Bejan, A., and Fautrelle, Y., 2003, "Constructal Multi-Scale Structure for Maximal Heat Transfer Density," Acta Mech., 163(1-2), pp. 39-49.

[16] Bejan, A., 2000, Shape and Structure, From Engineering to Nature, Cambridge University, Cambridge, UK

[17] Bejan, A., and Lorente, S., 2008, Design With Constructal Theory, Wiley, Hoboken, NJ.

[18] Barrau, J., Omri, M., Chemisana, D., Rosell, J., Ibañez, M., and Tadrist, L. 2012, "Numerical Study of a Hybrid Jet Impingement/Micro-Channel Cooling Scheme," Appl. Therm. Eng., 33-34, pp. 237-245.

[19] Sharma, C. S., Tiwari, M. K., Zimmermann, S., Brunschwiler, T., Schlottig, G, Michel, B., and Poulikakos, D., 2015, "Energy Efficient Hotspot-Targeted Embedded Liquid Cooling of Electronics," Appl. Energy, 138, pp. 414-422.

[20] "Spread Your Wings, It's Time to Fly," Last accessed July 26, 2006, www.nasa.gov

[21] Bejan, A., 1998, "Constructal Theory: From Thermodynamics and Geometric Optimization to Predicting the Shape in Nature," Energy Convers. Manage., 39(16-18), pp. 1705-1718.

[22] Bejan, A., and Lorente, S., 2010, "The Constructal Law and the Design of the Biosphere: Nature and Globalization," ASME J. Heat Transfer, 133(1), p. 011001.

[23] Reis, A. H., 2006, "Constructal View of Scaling Laws of River Basins," Geomorphology, 78(3-4), pp. 201-206.

[24] Miguel, A. F., 2006, "Constructal Pattern Formation in Stony Corals, Bacterial Colonies and Plant Roots Under Different Hydrodynamics Conditions," J. Theor. Biol., 242(4), pp. 954-961.

[25] Wechsatol, W., Ordonez, J. C., and Kosaraju, S., 2006, "Constructal Dendritic Geometry and the Existence of Asymmetric Bifurcation," J. Appl. Phys. 100(11), p. 113514

[26] Wang, X.-Q., Mujumdar, A. S., and Yap, C., 2005, "Numerical Analysis of Blockage and Optimization of Heat Transfer Performance of Fractal-Like Microchannel Nets," ASME J. Electron. Packag., 128(1), pp. 38-45.

[27] Azoumah, Y., Neveu, P., and Mazet, N., 2007, "Optimal Design of Thermochemical Reactors Based on Constructal Approach," AIChe J., 53(5), pp. $1257-1266$.

[28] Biserni, C., Rocha, L. A. O., Stanescu, G., and Lorenzini, E., 2007, "Constructal H-Shaped Cavities According to Bejan's Theory," Int. J. Heat Mass Transfer, 50(11-12), pp. 2132-2138.

[29] Lorenzini, G., Biserni, C., Garcia, F. L., and Rocha, L. A. O., 2012, "Geometric Optimization of a Convective T-Shaped Cavity on the Basis of Constructal Theory," Int. J. Heat Mass Transfer, 55(23-24), pp. 6951-6958.

[30] Lorenzini, G., Rocha, L. A. O., Biserni, C., dos Santos, E. D., and Isoldi, L. A., 2012, "Constructal Design of Cavities Inserted Into a Cylindrical Solid Body," ASME J. Heat Transfer, 134(7), p. 071301.

[31] Bejan, A., and Zane, J. P., 2012, Design in Nature, Doubleday, New York.

[32] Almogbel, M., and Bejan, A., 1999, "Conduction Trees With Spacings at the Tips," Int. J. Heat Mass Transfer, 42(20), pp. 3739-3756.

[33] Cetkin, E., 2014, "Three-Dimensional High Conductivity Trees for Volumetric Cooling,” Int. J. Energy Res., 38(12), pp. 1571-1577.

[34] Bejan, A., 2003, "Constructal Tree-Shaped Paths for Conduction and Convection,” Int. J. Energy Res., 27(4), pp. 283-299.

[35] Bejan, A., 1997, "Constructal-Theory Network of Conducting Paths for Cooling a Heat Generating Volume," Int. J. Heat Mass Transfer, 40(4), pp. 799-816.

[36] Rocha, L. A. O., Lorente, S., and Bejan, A., 2006, "Conduction Tree Networks With Loops for Cooling a Heat Generating Volume," Int. J. Heat Mass Transfer, 49(15-16), pp. 2626-2635.

[37] Ledezma, G. A., Bejan, A., and Errera, M. R., 1997, "Constructal Tree Networks for Heat Transfer," J. Appl. Phys., 82(1), pp. 89-100.

[38] Ledezma, G. A., and Bejan, A., 1998, "Constructal Three-Dimensional Trees for Conduction Between a Volume and One Point," ASME J. Heat Transfer, 120(4), pp. 977-984.

[39] Bhattacharjee, S., and Grosshandler, W. L., 1988, "The Formation of Wall Jet Near a High Temperature Wall Under Microgravity Environment," ASME National Heat Transfer Conference, Houston, TX, July 24-27, American Society of Mechanical Engineers, New York, Vol. 96, pp. 711-716.

[40] Petrescu, S., 1994, "Comments on "the Optimal Spacing of Parallel Plates Cooled by Forced Convection'," Int. J. Heat Mass Transfer, 37(8), p. 1283.

[41] "Comsol Multiphysics," www.comsol.com 